\title{
When is the category of flat modules abelian?
}

\author{
by
}

\section{J. L. García and J. Martínez Hernández (Murcia)}

\begin{abstract}
Let $\mathrm{Fl}(R)$ denote the category of flat right modules over an associative ring $R$. We find necessary and sufficient conditions for $\mathrm{Fl}(R)$ to be a Grothendieck category, in terms of properties of the ring $R$.
\end{abstract}

1. Introduction. Let $R$ be an associative ring with identity and denote by $\operatorname{Mod}(R)$ the category of all right $R$-modules, and by $\mathrm{Fl}(R)$ the full subcategory of $\operatorname{Mod}(R)$ whose objects are all the flat right $R$-modules. While, in general, $\mathrm{Fl}(R)$ need not be an abelian category, it might be so: for instance, let $A$ be a ring with a finite number of isomorphism classes of finitely presented indecomposable right modules, and such that every finitely presented right $A$-module is a direct sum of indecomposables, and consider then the direct sum $U_{A}$ of all the indecomposable finitely presented right $A$-modules (up to isomorphism). Then $U_{A}$ is finitely presented, and, if $R$ denotes the endomorphism ring $R=\operatorname{End}\left(U_{A}\right)$, then the category $\mathrm{Fl}(R)$ is equivalent to $\operatorname{Mod}(A)[15$, Corollary 2.9], so that it is abelian. In [10, p. 29], Jøndrup and Simson pointed out that it would be interesting to have a characterization of all rings $R$ such that $\mathrm{Fl}(R)$ is abelian, and they conjectured therein that all such rings $R$ having a decomposition into a direct sum of indecomposable right ideals are Morita equivalent to a ring of the form $\operatorname{End}\left(U_{A}\right)$ for $A$ and $U$ as above.

On the other hand, Tachikawa [17] studied in 1974 the rings $R$ such that the category $\mathcal{A}$ of all projective right $R$-modules is a Grothendieck category. He obtained two characterizations of such rings $R$ :

- They are the semiprimary and QF-3 rings $R$ such that the dominant dimension of $R_{R}$ is $\geq 2$, and the global dimension of $R$ is $\leq 2$.

- They are the semiprimary QF-3 rings $R$ which are the endomorphism rings $\operatorname{End}\left(U_{A}\right)$ of the direct sum of all the finitely generated indecomposable modules over a ring $A$ of finite representation type.

1991 Mathematics Subject Classification: 16D40, 16D90, 16 S90. 
Since the rings $R$ appearing in Tachikawa's paper are right perfect, the category $\mathcal{A}$ is, in that case, the same as $\operatorname{Fl}(R)$ and hence the problem in Jøndrup-Simson's paper may be viewed as a generalization of the problem solved by Tachikawa. Our aim in this paper is to answer the problem of Jøndrup and Simson, stating a characterization for the rings $R$ such that $\mathrm{Fl}(R)$ is an abelian category (see Theorem 3). As a consequence, we get Corollary 5, which asserts that the category $\operatorname{Fl}(R)$ over a (left and right) coherent ring is abelian if and only if $R$ is of weak global dimension $\leq 2$, and the flat-dominant dimension of $R_{R}$ is $\geq 2$. However, the conjecture stated in [10] which has been just mentioned remains open.

2. Main results. One important step in the solution is to consider the class of FTF-rings which was introduced by Gómez Torrecillas in [7]. So, we begin by recalling the definition of such rings, which are more fully investigated in [7] and [8].

Definition 1 ([7, p. 61], [8, p. 531]). A ring $R$ is a right FTF-ring if the class of right $R$-modules which are (isomorphic to) submodules of flat modules is the torsionfree class for a hereditary torsion theory $\tau$ of $\operatorname{Mod}(R)$.

From now on, if $R$ is assumed to be a right FTF-ring, then $\tau$ will denote the hereditary torsion theory of $\operatorname{Mod}(R)$ just defined. The following result can be gathered in [7, Chapter 2.4], but, for the sake of completeness, we indicate how the proof can be seen from results in [8]:

Proposition 2. If $R$ is a right perfect and right FTF-ring, then $R$ is $Q F$-3. In that case, $R$ is also semiprimary and $\tau$-artinian. Conversely, if $R$ is a perfect and QF-3 ring, then $R$ is right FTF.

Proof. If $R$ is right perfect and right FTF, then the same proof of [8, Corollary 2.11] works to show that $R$ is QF-3. Again, the proof of [8, Corollary 2.11] along with [8, Theorem 2.7] shows that, in that case, $R$ is also semiprimary and $\tau$-artinian. The converse follows from [8, Corollary 2.11].

Recall also from [12, p. 1060] that if $\sigma$ is a hereditary torsion theory of $\operatorname{Mod}(R)$, a right $R$-module $M_{R}$ is called $\sigma$-finitely generated if there is a submodule $L \subseteq M$ such that $L_{R}$ is finitely generated and $M / L$ is $\sigma$-torsion. A finitely generated module $M_{R}$ is said to be $\sigma$-finitely presented in case there exists a short exact sequence

$$
0 \rightarrow K \rightarrow P \rightarrow M \rightarrow 0
$$

in $\operatorname{Mod}(R)$ such that $P$ is finitely generated projective and $K$ is $\sigma$-finitely generated. 
In [11, Definition 1.2], a ring $R$ is called $\sigma$-coherent in case every finitely generated right ideal of $R$ is $\sigma$-finitely presented. By extending this definition, we will say that the ring $R$ is weakly $\sigma$-coherent if every finitely generated right ideal $I_{R}$ of $R$ such that $R / I$ is $\sigma$-torsion is $\sigma$-finitely presented.

Finally, we will say that the ring $R$ has flat-dominant dimension $\geq n$ if the first $n$ terms of a minimal injective resolution of $R_{R}$ are flat (see [9]).

We are now ready to state and prove our main result. In what follows, $\lambda$ will denote the Lambek torsion theory of $\operatorname{Mod}(R)$ (i.e., the $\lambda$-torsionfree modules are exactly the right $R$-modules cogenerated by the injective envelope of $R_{R}, E\left(R_{R}\right)$ ).

Theorem 3. Let $R$ be a ring and $\operatorname{Fl}(R)$ the full subcategory of $\operatorname{Mod}(R)$ consisting of all flat right $R$-modules. Then $\mathrm{Fl}(R)$ is an abelian category if and only the following three conditions are satisfied:

(a) $R$ is a right FTF-ring which is weakly $\lambda$-coherent;

(b) The weak global dimension $w D(R)$ of $R$ is at most 2 ;

(c) The flat-dominant dimension of $R_{R}$ is $\geq 2$.

Proof. Necessity. Assume that $\mathrm{Fl}(R)$ is abelian. Since the direct sum of a family of flat modules is flat, $\mathrm{Fl}(R)$ is a cocomplete abelian category having a generator, $R_{R}$. Moreover, since direct limits of flat modules are flat [16, Proposition I.10.3], one sees easily that direct limits are exact in $\mathrm{Fl}(R)$ and hence, the category $\mathrm{Fl}(R)$ is in fact a Grothendieck category [16, p. 114].

By applying the Gabriel-Popescu Theorem [16, Theorem X.4.1] to the generator $R_{R}$ of $\mathrm{Fl}(R)$ and bearing in mind that $\operatorname{Hom}_{\mathrm{Fl}(R)}(R, R) \cong R$, we deduce that the functor $F: \operatorname{Fl}(R) \rightarrow \operatorname{Mod}(R)$ with $F(X)=\operatorname{Hom}_{R}(R, X)$ $\cong X_{R}$ is full and faithful. Moreover, $F$ is naturally equivalent to the inclusion functor and establishes an equivalence between the category $\mathrm{Fl}(R)$ and the quotient category $\operatorname{Mod}(R, \mathcal{F})$ of $\operatorname{Mod}(R)$ whose $\mathcal{F}$-closed objects are precisely the flat modules. So, the class of $\mathcal{F}$-torsionfree modules coincides with the class of submodules of flat modules, so that $R$ is right FTF, by Definition 1, and $\mathcal{F}$ is the Gabriel topology corresponding to the torsion theory $\tau$.

By identifying the above functor $F$ and the inclusion functor from $\mathrm{Fl}(R)$ to $\operatorname{Mod}(R)$, we see that $\operatorname{Fl}(R)$ coincides with $\operatorname{Mod}(R, \mathcal{F})=\operatorname{Mod}(R, \tau)$, and thus $\operatorname{Fl}(R)$ is a Giraud subcategory of $\operatorname{Mod}(R)$ [16, Theorem X.2.1], from which it follows that the inclusion functor has an exact left adjoint $G: \operatorname{Mod}(R) \rightarrow \mathrm{Fl}(R)$. If $\Psi$ denotes the unit of the adjunction, there are homomorphisms $\Psi_{M}: M \rightarrow G(M)$ such that the kernel and the cokernel of $\Psi_{M}$ are $\tau$-torsion modules. $G(M)$ is obviously a flat module, and if $F_{R}$ is 
any flat module and we have a diagram

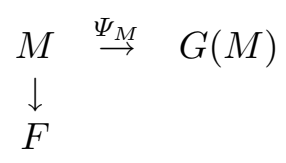

then the diagram can be completed commutatively by a homomorphism $G(M) \rightarrow F$ in a unique way. This implies that the homomorphism $\Psi_{M}$ : $M \rightarrow G(M)$ is a flat envelope of $M_{R}$ [4] which completes the diagrams in a unique way. It now follows from [1, Proposition 2.1] that $R$ is a left coherent ring and that the weak global dimension of $R, w D(R)$, is $\leq 2$. This proves (b).

Now, since the $\tau$-closed modules coincide with the flat modules, we see that the direct limit of $\tau$-closed modules of $\operatorname{Mod}(R)$ is always $\tau$-closed. By [16, Exercise XIII.1.2], $R$ is a finitely presented object of $\mathrm{Fl}(R)$. Now, let $I_{R}$ be a finitely generated right ideal of $R$ such that $R / I$ is $\lambda$-torsion. Then the left annihilator $l(I)=0$ [16, Proposition VI.6.4], and hence $R / I$ is also $\tau$-torsion (because homomorphisms from $R / I$ to flat modules have to factor through a projective module [7, Proposición 2.1.5]). Consider any exact sequence $0 \rightarrow K \rightarrow P \rightarrow I \rightarrow 0$ in $\operatorname{Mod}(R)$, with $P_{R}$ projective and finitely generated. If we apply the exact left adjoint $G: \operatorname{Mod}(R) \rightarrow$ $\mathrm{Fl}(R)$ of the inclusion functor, then we obtain the following exact sequence in the category $\mathrm{Fl}(R): 0 \rightarrow G(K) \rightarrow P \rightarrow R \rightarrow 0$. Since $R$ and $P$ are finitely presented in $\mathrm{Fl}(R)$, we deduce that $G(K)$ is finitely generated in $\mathrm{Fl}(R)=\operatorname{Mod}(R, \tau)$. But this implies that $K_{R}$ is $\tau$-finitely generated by [16, Proposition XIII.1.1]. Thus $K / L$ is $\tau$-torsion for some finitely generated submodule $L$ of $K$. Since $R$ is right FTF, $E\left(R_{R}\right)$ is flat by [8, Proposition 2.1] and hence $\operatorname{Hom}_{R}\left(K / L, E\left(R_{R}\right)\right)=0$. This means that $K / L$ is also $\lambda$-torsion and $I_{R}$ is $\lambda$-finitely presented. This completes the proof of condition (a) by showing that $R$ is weakly $\lambda$-coherent.

Finally, we have to consider the flat-dominant dimension of $R_{R}$ in order to prove condition (c). Let us look at the short exact sequence of $\operatorname{Mod}(R)$ :

$$
0 \rightarrow R_{R} \rightarrow E\left(R_{R}\right) \rightarrow Q \rightarrow 0
$$

where $E\left(R_{R}\right)$ is flat, as we saw above. Since both $R_{R}$ and $E\left(R_{R}\right)$ are $\tau$-closed modules, one may infer that $Q$ must be $\tau$-torsionfree [16, Proposition IX.4.2]. Hence, $Q$ is isomorphic to a submodule of a flat module and, therefore, to a submodule of an injective flat module. So, we have an injective resolution

$$
0 \rightarrow R_{R} \rightarrow E_{0} \rightarrow E_{1}
$$

where $E_{0}$ and $E_{1}$ are flat injective modules. This shows that the flatdominant dimension of $R_{R}$ is $\geq 2$.

Sufficiency. Since $R$ is a right FTF-ring, by Definition 1 there is a hereditary torsion theory $\tau$ of $\operatorname{Mod}(R)$ such that a module is $\tau$-torsionfree if 
and only if it can be embedded in a flat module. To show that $\operatorname{Fl}(R)$ is a Grothendieck category, it will be enough to see that a right $R$-module is flat if and only if it is $\tau$-closed [16, Theorem X.1.6].

We note first that all flat injective left $R$-modules are $\tau$-closed [16, Definition, p. 198]. Thus, condition (c) of the hypotheses implies that $R_{R}$ is a submodule of a $\tau$-closed module such that the quotient is $\tau$-torsionfree. But then $R_{R}$ is $\tau$-closed, by [16, Proposition IX.4.2]. As a consequence, every finitely generated projective right $R$-module is also $\tau$-closed.

Next we show that $R$ is a finitely presented object of the quotient category $\operatorname{Mod}(R, \tau)$. To this end, let $0 \rightarrow K \rightarrow M \rightarrow R \rightarrow 0$ be any short exact sequence in $\operatorname{Mod}(R, \tau)$, with $M$ finitely generated. By applying the inclusion functor from $\operatorname{Mod}(R, \tau)$ to $\operatorname{Mod}(R)$, we obtain another short exact sequence in $\operatorname{Mod}(R)$ :

$$
0 \rightarrow K \rightarrow M \rightarrow I \rightarrow 0
$$

and now $I_{R}$ is a right ideal of $R$ such that the localization of $I_{R}$ is $R_{R}$, so that in particular, $R / I$ is $\tau$-torsion. By using [16, Proposition XIII.1.1], we have a finitely generated submodule $N \subseteq M$ such that $M / N$ is $\tau$-torsion, and a new short exact sequence in $\operatorname{Mod}(\bar{R})$ :

$$
0 \rightarrow K \cap N \rightarrow N \rightarrow I^{\prime} \rightarrow 0
$$

where $I_{R}^{\prime}$ has the same properties as $I_{R}$, i.e., $R / I^{\prime}$ is $\tau$-torsion. Since $R$ is right FTF, $\operatorname{Hom}_{R}\left(R / I^{\prime}, E\left(R_{R}\right)\right)=0$, and thus $R / I^{\prime}$ is also $\lambda$-torsion. By condition (a), $R$ is weakly $\lambda$-coherent, so that $I_{R}^{\prime}$ is $\lambda$-finitely presented. Now, by [11, Lemma 2.4], there exists a short exact sequence in $\operatorname{Mod}(R)$,

$$
0 \rightarrow X \rightarrow Y \rightarrow I^{\prime} \rightarrow 0
$$

such that $Y$ is finitely presented and $X$ is $\lambda$-torsion. If $F_{R}$ is a flat right $R$-module and $E_{R}$ is its injective envelope, which is also flat by [8, Proposition 2.1], then any homomorphism $g: X \rightarrow F$ could be extended to a homomorphism $h: Y \rightarrow E$. Since $E_{R}$ is flat, $h$ factors through a finitely generated projective, and therefore so does $g$ followed by the inclusion $F \rightarrow E$. But then $g$ must be 0 , as $\operatorname{Hom}_{R}(X, R)=0$. This implies that $X$ is also $\tau$-torsion, and hence $I^{\prime}$ has to be $\tau$-finitely presented. By [11, Proposition 2.3], $K \cap N$ is $\tau$-finitely generated and $K /(K \cap N)$ is $\tau$ torsion, because $M / N$ is. Consequently, $K$ is the $\tau$-localization of a finitely generated submodule $L$ of $K \cap N$. But this implies that there exists an epimorphism $\varepsilon: P \rightarrow L$ with $P_{R}$ finitely generated and projective, and hence $\tau$-closed. This epimorphism induces, by localization, an epimorphism in $\operatorname{Mod}(R, \tau), P \rightarrow K$, which shows that $K$ is a finitely generated object of $\operatorname{Mod}(R, \tau)$. Thus, $R$ is finitely presented in $\operatorname{Mod}(R, \tau)$. By [16, Exercise XIII.1.2], every direct limit of $\tau$-closed modules is $\tau$-closed. Since every flat 
module is a direct limit of finitely generated projective modules and these are $\tau$-closed, we deduce that every flat module is $\tau$-closed.

It remains to show that every $\tau$-closed module $M_{R}$ is a flat module. By the definition of $\tau, M_{R}$ has to be a submodule of a flat module $F_{R}$, so that we get an exact sequence

$$
0 \rightarrow M \rightarrow F \rightarrow N \rightarrow 0
$$

where $N_{R}$ is also $\tau$-torsionfree [16, Proposition IX.4.2]. But this implies that $N_{R}$ is a submodule of a flat module $F_{R}^{\prime}$, which gives the exact sequence

$$
0 \rightarrow M \rightarrow F \rightarrow F^{\prime} \rightarrow L \rightarrow 0 .
$$

The fact that the weak global dimension of $R$ is $\leq 2$ now implies that $M_{R}$ is indeed flat, as was to be shown.

We now remark that the first characterization in [17] of rings $R$ for which the class of projective modules is a Grothendieck category may be found as a consequence of Theorem 3 above. Indeed, we can easily show the following:

Corollary 4 (Tachikawa, 1974). The category $\mathcal{A}$ of all projective right modules over a ring $R$ is a Grothendieck category if and only if $R$ is a perfect and QF-3 ring such that the global dimension of $R$ is at most 2, and the dominant dimension of $R_{R}$ is $\geq 2$.

Pro of. Suppose that $R$ satisfies the conditions of the statement. Since $R$ is perfect, $\mathcal{A}=\operatorname{Fl}(R)$, so that it is enough to see that $R$ satisfies conditions (a), (b), (c) of Theorem 3. But, by Proposition 2, $R$ is right FTF and $\tau$-artinian, hence it is clearly weakly $\tau$-coherent and weakly $\lambda$-coherent, so that $R$ satisfies (a). Conditions (b) and (c) are immediate from the other hypotheses.

Assume now that $\mathcal{A}$ is Grothendieck. Then it is easy to see, as in [17], that $R$ is right perfect. Thus, $\mathrm{Fl}(R)$ is a Grothendieck category and $R$ satisfies conditions (a), (b), (c) of Theorem 3. By (a) and Proposition 2, $R$ is QF-3. Then (b) and (c) imply the rest of the conditions in the corollary.

We next show the following corollaries.

COROLlaRY 5. Let $R$ be left and right coherent. Then $\mathrm{Fl}(R)$ is an abelian category if and only if $w D(R) \leq 2$ and the flat-dominant dimension of $R_{R}$ is $\geq 2$.

Proof. The conditions are necessary by Theorem 3. Conversely, if they hold, then $R$ is right FTF, by [8, Proposition 2.2], and it is obviously $\lambda$-coherent, since it is right coherent. By Theorem $3, \mathrm{Fl}(R)$ is abelian.

COROLlary 6. Let $R$ be left and right noetherian. Then $\mathrm{Fl}(R)$ is an abelian category if and only if $\mathrm{Fl}\left(R^{\mathrm{op}}\right)$ is also an abelian category. This happens if and only if $R$ is an Auslander ring (see [9]). 
Pr o of. By [8, Proposition 2.3], the ring $R^{\text {op }}$ is also left FTF, provided $\mathrm{Fl}(R)$ is abelian. Of course, $w D\left(R^{\mathrm{op}}\right) \leq 2$, by the hypothesis. Finally, the flat-dominant dimension of $R^{\text {op }}$ is $\geq 2$ in view of [9, Theorem]. By Theorem 3 , the category $\mathrm{Fl}\left(R^{\mathrm{op}}\right)$ is abelian.

Recall from [3] that a ring $R$ is said to be a right IF-ring in case each injective right $R$-module is flat. The following characterization is immediate.

COROLlaRY 7. Let $R$ be a right IF-ring. Then $\mathrm{Fl}(R)$ is an abelian category if and only if $R$ is a (von Neumann) regular ring.

$\operatorname{Pro}$ of. If $\operatorname{Fl}(R)$ is abelian, then by Theorem $3, R$ has finite weak global dimension. By [3, Proposition 5], $R$ has to be regular.

COROllary 8. Let $R$ be a commutative ring. Then $\operatorname{Fl}(R)$ is abelian if and only if $R$ is a (von Neumann) regular ring.

Proof. Assume first that $R$ is a local ring. Then, by Theorem $3, w D(R)$ $\leq 2$ (and $R$ is coherent, by the proof of the Theorem), and from [18, Corollary 5.16], we see that $R$ is a domain. The injective envelope of $R$ is its field of quotients, $Q$, and, since the flat-dominant dimension of $R$ has to be $\geq 2$, we see that $Q / R$ must be torsion and torsionfree, from which it follows that $R=Q$ is a field.

Consider now the general case of a commutative ring $R$ such that $\mathrm{Fl}(R)$ is a Grothendieck category. Let $\mathfrak{p}$ be a prime ideal of $R$. The localization functor $b: \operatorname{Mod}(R) \rightarrow \operatorname{Mod}\left(R_{\mathfrak{p}}\right)$ induces a functor $b^{\prime}: \operatorname{Fl}(R) \rightarrow \operatorname{Fl}\left(R_{\mathfrak{p}}\right)$, which preserves kernels. Furthermore, $b^{\prime}$ is a left adjoint of the inclusion functor from $\mathrm{Fl}\left(R_{\mathfrak{p}}\right)$ to $\mathrm{Fl}(R)$. Hence, $\mathrm{Fl}\left(R_{\mathfrak{p}}\right)$ is an abelian category by [16, Proposition X.1.3]. Now, by the first part of the proof, $R_{\mathfrak{p}}$ is a field. We deduce finally that $R$ is (von Neumann) regular, from [13, Lemma 8 and Theorem 6].

Finally, we give an example of an indecomposable ring $R$ which is neither regular nor perfect and for which $\mathrm{Fl}(R)$ is abelian, thus exhibiting a case not included in Tachikawa's theorem.

EXAMPLE. We start with a field $k$ and a finite-dimensional $k$-algebra $A$ with a connected Gabriel quiver such that $A$ is not of finite representation type. By results in [2], $A$ is not right pure-semisimple (that is, it is not the case that every right $A$-module is a direct sum of finitely generated indecomposable modules). Let $\left\{U_{\lambda}\right\}_{\lambda \in \Lambda}$ be the family of all isomorphism classes of finitely presented indecomposable right $A$-modules, and take $T$ as Gabriel functor ring, i.e., $T=\bigoplus_{\Lambda} \operatorname{Hom}_{A}\left(U_{\lambda}, U_{\mu}\right)$ with the obvious multiplication. We remark that $T$ is a ring with enough idempotents [6, p. 138], but without an identity. We denote by $\operatorname{Mod}(T)$ the category of all unitary right $T$-modules. $T$ is also a $k$-algebra in a natural way, and we know that there 
is an equivalence between the category $\operatorname{Mod}(A)$ and the category $\operatorname{Fl}(T)$ of all right flat (and unitary) $T$-modules (see [15]). Since $A$ is not right puresemisimple, $T$ is not right perfect (by [14] and [5, Theorem]). Also, $\mathrm{Fl}(T)$ does not coincide with the category $\operatorname{Mod}(T)$, because this would imply that $A$ is semisimple.

Define now the ring $R=T \times k$, with multiplication given by

$$
(t, \alpha) \cdot(s, \beta)=(t s+t \beta+s \alpha, \alpha \beta) .
$$

Note that $T$ is a twosided ideal of $R$ satisfying the conditions of [16, Proposition XI.3.13] on both sides, with $k \cong R / T$. Hence, all right $k$-modules are flat as right $R$-modules, and, in particular, for every right $R$-module $M_{R}$, $M / M T$ is always a flat right $R$-module. This implies that $M T$ is a pure submodule of $M_{R}$, so that $M_{R}$ is flat if and only if $M T$ is flat. On the other hand, it is clear that the category $\operatorname{Mod}(T)$ may be identified with the full subcategory of $\operatorname{Mod}(R)$ consisting of all right $R$-modules $M$ such that $M=M T$, and this full subcategory is closed under submodules, quotient modules, direct sums, extensions and direct limits. Also, a right $R$-module $M$ is projective if and only if $M T$ is projective as a right $T$-module. Thus, the condition that $M_{R}$ be flat is equivalent to $M T$ being flat in the category $\operatorname{Mod}(T)$.

Let us denote by $\mathcal{T}$ the class of right $T$-modules $X$ satisfying $\operatorname{Hom}_{T}(X, F)$ $=0$ for every flat right $T$-module $F$. Then $\mathcal{T}$ is a hereditary torsion class in $\operatorname{Mod}(T)$. Define now the class $\mathcal{T}^{\prime}$ exactly in the same way but in the category $\operatorname{Mod}(R)$. Then we easily see that $\mathcal{T}^{\prime}$ is contained in $\operatorname{Mod}(T)$ and, in fact, coincides with $\mathcal{T}$ - up to the obvious identification of $\operatorname{Mod}(T)$ as a full subcategory of $\operatorname{Mod}(R)$. Therefore, $\mathcal{T}^{\prime}$ is a hereditary torsion class in $\operatorname{Mod}(R)$ and hence $R$ is right FTF. It is clear that $w D(R) \leq 2$, from which it follows that every $\mathcal{T}^{\prime}$-closed right $R$-module is flat. For the converse, note that for each $M_{R}$, the injective envelope of $M T$ in $\operatorname{Mod}(T)$ is just $E(M) T$. Since every flat module in $\operatorname{Mod}(T)$ is $\mathcal{T}$-closed, it follows that if $M_{R}$ is flat, then it is also $\mathcal{T}^{\prime}$-closed. This shows that $\mathrm{Fl}(R)$ is a Grothendieck category.

On the other hand, $R$ is not von Neumann regular, because in that case, every right unitary $T$-module would be flat. Also, $R$ is not right perfect, because $T$ is not right perfect. Finally, $R$ has no nontrivial central idempotents, because the quiver of $A$ is connected.

We finish with the following

Problem. Is the condition that $R$ be weakly $\lambda$-coherent in Theorem 3 a consequence of the other hypotheses?

Acknowledgements. The authors want to thank Professor Daniel Simson for having suggested the problem studied in this paper, and for many helpful remarks and comments about the subject. They also acknowledge financial support from the D.G.I.C.Y.T. (PB90-0300-C02-02). 


\section{References}

[1] J. Asensio Mayor and J. Martínez Hernández, On flat and projective envelopes, J. Algebra 160 (1993), 434-440.

[2] M. Auslander, Large modules over artin algebras, in: Algebra, Topology and Category Theory, Academic Press, 1976, 3-17.

[3] R. R. Colby, Rings which have flat injective modules, J. Algebra 35 (1975), 239252.

[4] E. E. Enochs, Injective and flat covers, envelopes and resolvents, Israel J. Math. 39 (1981), 189-209.

[5] K. R. Fuller, On rings whose left modules are direct sums of finitely generated modules, Proc. Amer. Math. Soc. 54 (1976), 39-44.

[6] J. L. García and J. Martínez Hernández, Purity through Gabriel's functor rings, Bull. Soc. Math. Belgique 45 (1993), 137-152.

[7] J. Gómez Torrecillas, Anillos con módulos planos libres de torsión, Ph.D. Thesis, University of Granada, 1992.

[8] J. Gómez Torrecillas and B. Torrecillas, Flat torsionfree modules and QF-3 rings, Osaka J. Math. 30 (1993), 529-542.

[9] M. Hoshino, On dominant dimension of Noetherian rings, Osaka J. Math. 26 (1989), 275-280.

[10] S. Jøndrup and D. Simson, Indecomposable modules over semiperfect rings, J. Algebra 73 (1981), 23-29.

[11] M. F. Jones, Coherence relative to an hereditary torsion theory, Comm. Algebra 10 (1982), 719-739.

[12] R. W. Miller and M. L. Teply, On flatness relative to a torsion theory, ibid. 6 (1978), 1037-1071.

[13] A. Rosenberg and D. Zelinsky, Finiteness of the injective hull, Math. Z. 70 (1959), 372-380.

[14] D. Simson, Functor categories in which every flat object is projective, Bull. Acad. Polon. Sci. 22 (1974), 375-380.

[15] - On pure global dimension of locally finitely presented Grothendieck categories, Fund. Math. 96 (1977), 91-116.

[16] B. Stenström, Rings of Quotients, Springer, Berlin, 1975.

[17] H. Tachikawa, QF-3 rings and categories of projective modules, J. Algebra 28 (1974), 408-413.

[18] W. V. Vasconcelos, The Rings of Dimension Two, Marcel Dekker, New York, 1975.

DEPARTAMENTO DE MATEMÁTICAS

UNIVERSIDAD DE MURCIA

30071 MURCIA, SPAIN 